\title{
Tendências de índices de extremos climáticos para a região de Manaus-AM
}

\author{
Carlos Antonio Costa dos SANTOS ${ }^{1}$, Prakki SATYAMURTY², Edilanê Mendes dos SANTOS³ \\ RESUMO \\ Foram estudadas tendências nos índices de extremos climáticos baseados em dados de precipitação para três estaçóes localizadas \\ em Manaus e na regiấo circunvizinha para o período de 1971-2007. Baseado nos resultados obtidos pode ser observado que \\ houve aumento da precipitação total anual sobre a regiáo estudada. A estaçáo localizada na zona urbana de Manaus (INMET) \\ teve aumento, com significância estatística, dos eventos de precipitaçáo iguais ou superiores a $50 \mathrm{~mm}(\mathrm{R} 50 \mathrm{~mm})$, na precipitação \\ máxima acumulada em cinco dias consecutivos (Rx5day) e nos dias úmidos (R95p), indicando que Manaus poderá sofrer com \\ o aumento das chuvas extremas. Aumento das anomalias positivas de TSM no Oceano Pacífico Equatorial leva a um aumento \\ dos dias consecutivos secos e diminuiçáo dos eventos extremos de chuva e da precipitaçáo total sobre a bacia amazônica. As \\ anomalias positivas de TSM no Oceano Atlântico Sul induz o deslocamento da ZCIT mais ao sul do Equador levando a um \\ aumento da precipitaçáo sobre a regiáo de Manaus. Os resultados obtidos aqui têm potencial para possíveis previsóes das \\ características da precipitação em Manaus.
}

PaLaVRAS-CHAVE: Precipitação, El Niño, Temperatura da Superfície do Mar, TNAI, TSAI.

\section{Trends of extreme climatic indices for Manaus - AM region}

\section{ABSTRACT}

Trends in the extreme climate indices based on precipitation data at three stations in and around the city of Manaus for the period 1971-2007 are studied. Based on the results obtained it can be observed that there was an increase of the annual total precipitation in the area studied. At the station located in the city of Manaus (INMET), the frequency of precipitation events with $50 \mathrm{~mm}(\mathrm{R} 50 \mathrm{~mm})$ or more, the amount precipitation in five consecutive days (Rx5day) and the number of wet days (R95p), showed an increase, with statistical significance, indicating that Manaus may suffer with the increase of the extreme rainfall events. An increase in the positive SST anomalies in the Equatorial Pacific Ocean induce an increase of the consecutive dry days and decrease of the extreme rainfall events and, consequently, in the total precipitation in the Amazon basin. The positive SST anomalies in the South Atlantic Ocean induce the displacement of ITCZ to the south of Equator causing an increase of the precipitation in the Manaus region. The results obtained here have potential for possible prediction of precipitation characteristics at Manaus.

KEYWORDS: Precipitation, El Niño, Sea Surface Temperature, TNAI, TSAI.

\footnotetext{
1 Universidade Federal de Campina Grande, UACA, Av. Aprígio Veloso, 882, Bodocongó, 58109-970 - Campina Grande, PB - Brasil. Fone/fax: 8321011202 , E-mail: carlos@dca.ufcg.edu.br; carlostorm@gmail.com;

2 Universidade do Estado do Amazonas, CESTU, Avenida Djalma Batista 3578, Flores. CEP 69077-000, Manaus, AM - Brasil. E-mail: saty.prakki@gmail.com;

3 Programa de Pós-Graduação em Clima e Ambiente, Instituto Nacional de Pesquisas da Amazônia, Avenida André Araújo, 2936, Aleixo, CEP 69060-001 Manaus, AM, Brasil. E-mail: edilanemendes@bol.com.br.
} 


\section{INTRODUÇÃO}

Estudos recentes apontam que o aquecimento global trará impactos potencialmente catastróficos para a Amazônia, ao passo que a manutençáo da floresta amazônica oferece uma das opçôes mais valiosas e de baixo custo para mitigar as mudanças climáticas (Fearnside 2009). Sabe-se que o fenômeno de El Niño - Oscilação Sul (ENOS), causado por oscilaçôes da temperatura da superfície da água no Oceano Pacífico, assim como, as oscilaçôes da temperatura no Oceano Atlântico podem trazer impactos sobre a Amazônia, tais como secas e incêndios florestais. Tem sido observado também que as árvores amazônicas são sensíveis à ação do fogo e de estresse hídrico sob condiçôes quentes e secas. Ademais, a água reciclada pela floresta fornece parte da chuva que mantém as condiçóes climáticas apropriadas para floresta tropical, especialmente durante a estação seca (Fearnside 2009; Yoon e Zeng 2010).

O aquecimento global tem o potencial de causar altas taxas de evaporaçáo e intensificar o transporte de grandes quantidades de vapor de água na atmosfera, provavelmente acelerando o ciclo hidrológico global (Zhang et al. 2010). Uma das mais significativas consequências do aquecimento global poderá ser um aumento na magnitude e frequência dos extremos de precipitação através do acréscimo dos níveis de umidade na atmosfera e/ou atividades convectivas de grande escala (Shouraseni e Robert 2004).

Nas últimas décadas, as mudanças nos extremos climáticos têm atraído a atenção mundial porque os eventos climáticos extremos impactam os sistemas naturais e humanos mais que seus valores médios (Aguilar et al. 2009). A infra-estrutura social (abastecimento de água, por exemplo) é mais sensível aos eventos extremos. De acordo com o IPCC (2007), as mudanças na distribuição de plantas e animais selvagens, extinçóes induzidas pelo clima, mudanças na fenologia e na diversidade de espécies têm apresentado taxas de aumento.

Extremos de temperatura e precipitação têm sido estudados em escala global, nacional e regional. Em escala global, análises mais compreensivas dos extremos climáticos são discutidas no Quarto Relatório de Avaliação do IPCC (IPCC 2007). Na escala nacional e regional, têm sido desenvolvidos estudos das mudanças e seus impactos na Ásia, Pacífico Sul, Caribe, África, América do Sul e América do Norte. Existem marcantes consistências entre os resultados obtidos por estes estudos em termos de temperatura, mas menos coerência espacial nos extremos de precipitaçâo (You et al. 2010; Santos et al. 2011). No final da década de 1990 vários workshops desenvolveram índices para analisar extremos climáticos. Com o objetivo de criar um conjunto de índices que poderiam ser calculados para uma variedade de climas e possibilitar a comparação entre regiôes. Como resultados, 27 índices de mudanças climáticas, derivados de dados diários de temperatura e precipitaçáo, foram identificados (Zin et al. 2010).

O objetivo desse estudo é analisar as tendências dos índices de extremos climáticos, dependentes da precipitação pluvial diária, definidos pela Organizaçáo Meteorológica Mundial (Zhang e Yang 2004), para a cidade de Manaus-AM, assim como, analisar suas relaçóes com as anomalias da Temperatura da Superfície do Mar (TSM) nos Oceanos Pacífico (ENOS) e Attântico (Dipolo do Atlântico). Esse trabalho é de grande importância para a populaçấo da cidade de Manaus-AM, uma vez que alteraçóes no clima poderão influenciar os setores sócio-econômicos e causar impactos ambientais.

\section{MATERIAL E MÉTODOS}

Neste trabalho foram utilizados dados diários de precipitaçáo pluvial, para o período de 1971 a 2007, obtidos do Centro de Pesquisa Agroflorestal da Amazônia - EMBRAPA (2०52'S; 59059'W) situado a $30 \mathrm{~km}$ ao norte da cidade de Manaus, da Reserva Florestal Ducke (2055'S, $\left.59^{\circ} 57^{\prime} \mathrm{W}\right)$, área de proteção ambiental pertencente ao Instituto Nacional de Pesquisas da Amazônia - INPA, localizada a 25 $\mathrm{km}$ a nordeste da cidade de Manaus e da estação meteorológica do Instituto Nacional de Meteorologia - INMET (312'S, $59^{\circ} 95^{\prime} \mathrm{W}$ ) (Figura 1). Dados das anomalias mensais de TSM nas regiôes dos Oceanos Pacífico e Atlântico foram obtidos do site eletrônico do Climate Prediction Center (CPC) da National Oceanic and Atmospheric Administration (NOAA).

Os onze índices de eventos climáticos extremos derivados de dados de precipitação, utilizados nessa pesquisa, juntamente com suas definiçôes conforme o Expert Team on Climate Change Detection Monitoring and Indices (ETCCDMI), estấo mostrados na Tabela 1 . Na avaliação da homogeneização dos dados e no cálculo dos índices foi utilizada a metodologia de Zhang et al. (2005) implementada no software RClimdex

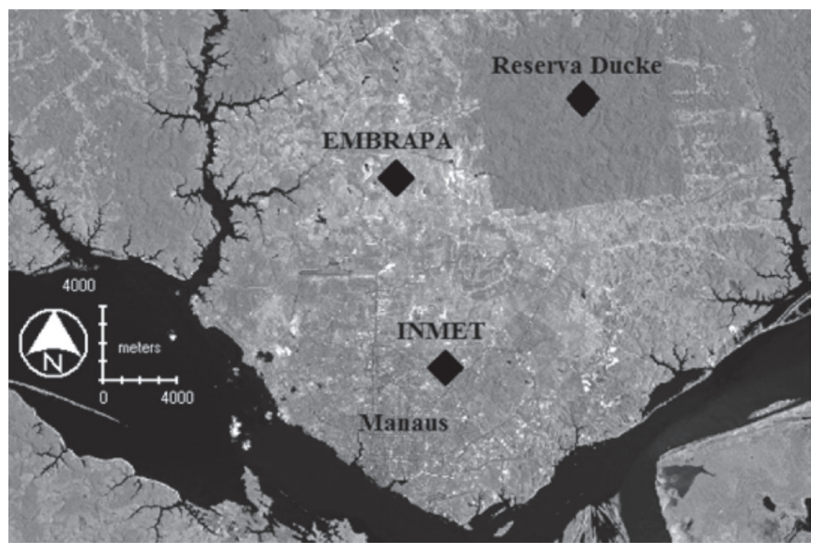

Figura 1 - Localização dos postos de coleta de dados de temperatura. 
Tabela 1 - Definição dos índices de extremos climáticos baseados em dados de precipitação utilizados nesse estudo

\begin{tabular}{|c|c|c|}
\hline Índices & Definição & Unidades \\
\hline Rx1day & Máxima precipitação em 1 dia & $\mathrm{mm}$ \\
\hline Rx5day & Máxima precipitação em 5 dias consecutivos & $\mathrm{mm}$ \\
\hline SDII & $\begin{array}{l}\text { Média anual da precipitação quando } \\
\text { PRCP } \geq 1,0 \mathrm{~mm}\end{array}$ & $\mathrm{~mm}$ \\
\hline R10mm & Número de dias em um ano quando $P R C P \geq 10 \mathrm{~mm}$ & dias \\
\hline $\mathrm{R} 20 \mathrm{~mm}$ & Número de dias em um ano quando $P R C P \geq 20 \mathrm{~mm}$ & dias \\
\hline R50mm & Número de dias em um ano quando $P R C P \geq 50 \mathrm{~mm}$ & dias \\
\hline DCS & $\begin{array}{l}\text { Máximo número de dias consecutivos secos com } \\
\text { PRCP }<1 \mathrm{~mm}\end{array}$ & dias \\
\hline DCU & $\begin{array}{l}\text { Máximo número de dias consecutivos úmidos } \\
\text { com PRCP } \geq 1 \mathrm{~mm}\end{array}$ & dias \\
\hline R95p & Dias úmidos quando $P R C P>95 p$ & $\mathrm{~mm}$ \\
\hline R99p & Dias extremamente úmidos quando $\mathrm{PRCP}>99 p$ & $\mathrm{~mm}$ \\
\hline PRCPTOT & Precipitação Total Anual quando (PRCP $\geq 1 \mathrm{~mm}$ ) & $\mathrm{mm}$ \\
\hline
\end{tabular}

1.9.0, desenvolvido e mantido pelos pesquisadores Xuebin Zhang e Feng Yang do Serviço de Meteorologia do Canadá. O controle de qualidade foi identificar erros nos dados, conforme proposto por Alexander et al. (2006), e implementado por Viney e Bates (2004). O controle de qualidade dos dados é um pré-requisito para o cálculo dos índices. No RClimdex obedece os seguintes procedimentos: 1) substitui todos os dados faltosos (codificados como -99.9) em um formato interno reconhecido pelo $\mathrm{R}$ e 2) substitui todos os valores não aceitáveis por -99.9. Estes valores incluem, por exemplo, quantidades de precipitação diárias menores que zero e, b) temperatura máxima diária menor que a temperatura mínima diária. Adicionalmente, o controle de qualidade também identifica valores extremos nas temperaturas diárias máximas e mínimas. Os valores extremos são valores diários que se encontram fora de um intervalo definido pelo usuário. Atualmente, este intervalo se define como $n$ vezes o desvio padrão do valor do dia, isto é, (média - $n^{*}$ std, média + $n^{*}$ std). Onde $s t d$ representa o desvio padráo para o dia e $n$ é uma entrada do usuário, nesse estudo foi aplicado $n=4$, que é o padráo adotado mundialmente (Zhang et al. 2005). Como o objetivo geral da pesquisa é identificar possíveis mudanças climáticas para a regiáo de Manaus utilizando os índices dependentes da precipitaçấo pluvial, entáo, o controle de qualidade referente aos dados de temperatura não foi executado. Assim como, no período estudado não foram encontrados problemas no referido procedimento de controle de qualidade.

O software fornece, para todos os índices, dados estatísticos, tais como: tendência linear anual calculada pelo método de mínimos quadrados; nível de significância estatística da tendência (valor p); coeficiente de determinação $\left(r^{2}\right)$ e erro padrão de estimativa; assim como os gráficos das séries anuais. Nesse estudo só foram consideradas significativas as tendências, que apresentam nível de significância de 95\% $(\mathrm{p}<0,05)$, conforme mostrado na Tabela 2 .

Estudos têm mostrado uma dependência entre a precipitaçâo na Amazônia com as anomalias de TSM dos Oceanos Pacífico Equatorial e Atlântico Tropical (Yoon e Zeng 2010). Logo, os índices de extremos climáticos anuais foram correlacionados com as anomalias anuais de TSM dos referidos oceanos. As anomalias anuais de TSM foram obtidas das áreas conhecidas por Niño 1+2, Niño 3, Niño 3.4 e Niño 4 (Menezes et al. 2008) no Oceano Pacífico Equatorial, e das regiôes de Índice do Atlântico Tropical Norte - TNAI (Tropical North Atlantic Index) e de Índice do Atlântico Tropical Sul - TSAI (Tropical South Atlantic Index) (Menezes et al. 2008) no Oceano Atlântico Tropical, foram utilizados valores médios anuais representativos para essas áreas. Com o intuito de analisar o comportamento das anomalias anuais de TSM nos Oceanos e suas influências sobre os índices de extremos climáticos anuais para a cidade de Manaus-AM, foi utilizado o método de Pearson na obtenção da correlação entre as anomalias anuais de TSM, no período de 1971 a 2007, e os índices de extremos climáticos anuais obtidos. Os índices foram correlacionados individualmente com a anomalia de TSM de cada área estudada e as correlaçôes, com significância estatística ao nível de 95\%, estão apresentadas na Tabela 3. A significância estatística foi obtida através do teste $t$-Student aplicada a uma série que apresenta graus de liberdade $(\mathrm{N})$ igual a 37, correspondentes aos 37 anos da série histórica de dados analisados.

Tabela 2 - Tendências anuais dos índices de extremos climáticos dependentes da precipitação para as três localidades estudadas.

\begin{tabular}{cccc}
\hline ÍNDICES & EMBRAPA & DUCKE & INMET \\
\hline Rx1day & 0.425 & 0.089 & 0.76 \\
\hline Rx5day & 0.304 & 0.23 & $1.459^{*}$ \\
\hline SDII & $0.046^{*}$ & $0.046^{*}$ & $0.056^{*}$ \\
\hline R10mm & $0.462^{*}$ & $0.294^{*}$ & 0.189 \\
\hline R20mm & $0.294^{*}$ & $0.205^{*}$ & 0.088 \\
\hline R50mm & -0.005 & 0.018 & $0.082^{*}$ \\
\hline DCS & 0.052 & 0.032 & -0.231 \\
\hline DCU & -0.019 & -0.027 & 0.007 \\
\hline R95p & 2.778 & 1.515 & $6.324^{*}$ \\
R99p & 0.172 & 1.047 & 2.484 \\
\hline PRCPTOT & $11.469^{*}$ & 7.423 & 7.671 \\
\hline
\end{tabular}

* Indica que o valor da tendência é significativo ao nível de significância de 95\% $(p<0,05)$. 


\section{RESULTADOS E DISCUSSÃO}

A colonização da Amazônia foi intensificada no período compreendido entre 1840 e 1910 culminando no rápido desenvolvimento urbano da cidade de Manaus. No entanto, somente a partir da implantação da Zona Franca de Manaus, na década de 1970, a cidade de Manaus apresentou um crescimento acelerado saindo de uma população de 284.000 habitantes em 1970, para 2.042.185 habitantes em 2009 (IBGE 2009). Esse crescimento levou a uma ocupação desordenada do espaço, apresentando hoje sérios problemas ambientais e sociais, entre eles estão o desmatamento e a construção de habitaçôes em áreas de risco de desabamentos e inundaçóes. Além disso, uma grande quantidade de gases de efeito estufa é emitida para a atmosfera, principalmente, pelo uso de veículos motorizados. Com isso, torna-se evidente a necessidade de estudar as tendências dos índices de extremos climáticos para a região de Manaus, uma vez que os mesmos podem ser utilizados como indicadores para prevenir ou mitigar desastres.

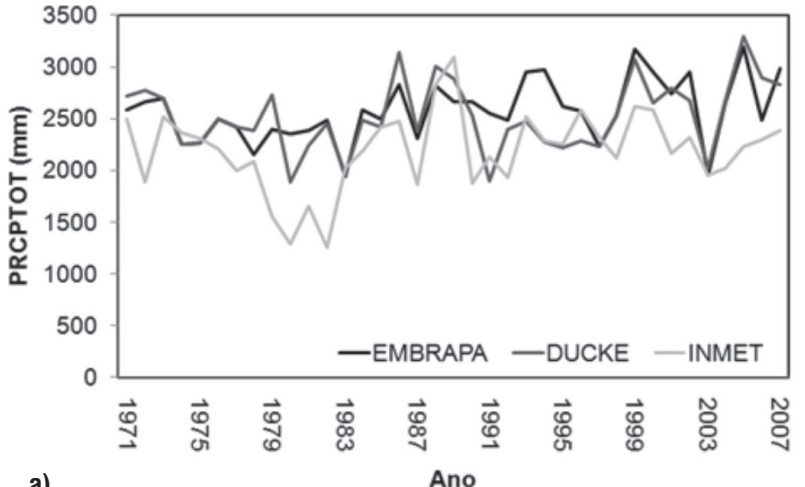

a)

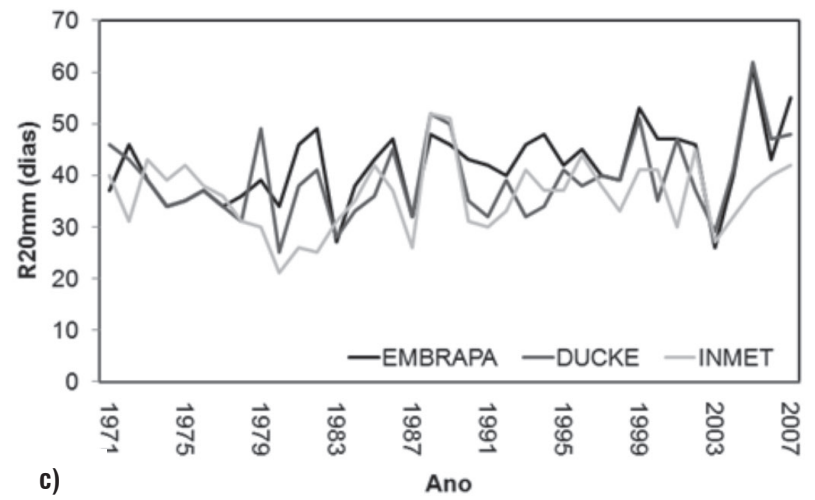

A Tabela 2 apresenta os valores das tendências anuais dos índices de extremos climáticos dependentes da precipitação para as três estaçóes meteorológicas instaladas nas proximidades da zona urbana de Manaus (EMBRAPA e Ducke) e na cidade de Manaus (INMET). Somente os valores destacados com o asterisco apresentam significância estatística ao nível de 95\%. É possível identificar um aumento com significância estatística da precipitação total anual, representada pelo índice PRCPTOT (Figura 2a). Esse índice mostra um aumento de aproximadamente $11,5 \mathrm{~mm}^{2} \mathrm{ano}^{-1}$ quando calculados com os dados medidos na estação meteorológica da EMBRAPA e, um aumento da ordem de 7,4 e 7,7 mm ano ${ }^{-1}$, sem significância estatística, para as estaçóes meteorológicas da Reserva Ducke e do INMET, respectivamente. Esses resultados indicam, mesmo com apenas uma estação apresentando significância estatística, um aumento predominante da precipitação total anual, o que pode trazer problemas para a população urbana de Manaus.
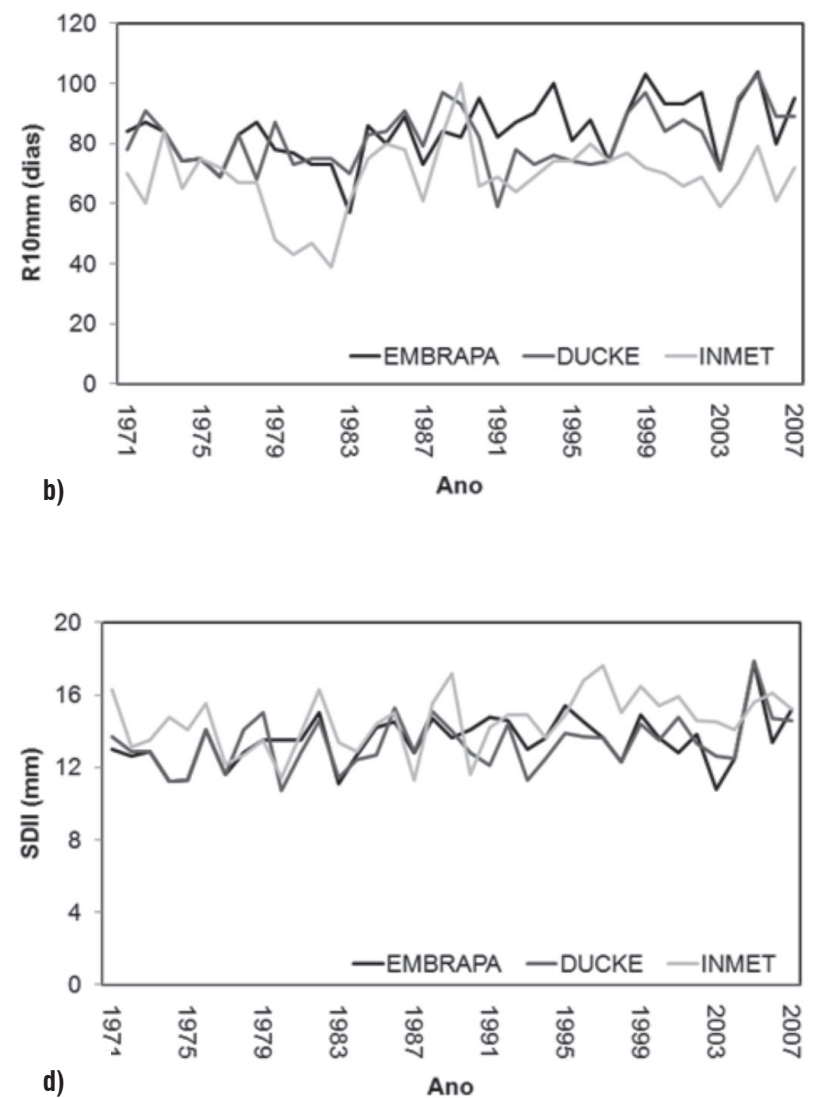

Figura 2 - Distribuição temporal dos índices R10mm (número de dias em um ano quando a precipitação é $\geq 10 \mathrm{~mm}$ ), R20mm (número de dias em um ano quando a precipitação é $\geq 20 \mathrm{~mm}$ ), SDII (média anual da precipitação) e PRCPTOT (precipitação total anual), para as três localidades estudadas (EMBRAPA, DUCKE e INMET) no período de 1971 a 2007. 
Os índices que representam o número de dias no ano com precipitaçâo superior a $10 \mathrm{~mm}$ e $20 \mathrm{~mm}(\mathrm{R} 10 \mathrm{~mm}$ e $\mathrm{R} 20 \mathrm{~mm}$ ), respectivamente, apresentaram aumento com significância estatística para as estaçóes meteorológicas instaladas na EMBRAPA e na Reserva Ducke, e aumento sem significância estatística para a estação do INMET (Figuras $2 \mathrm{~b}$ e $2 \mathrm{c}$ ), indicando um aumento no número de dias no ano com precipitaçóes igual ou superior aos referidos limiares (precipitaçôes intensas). Precipitaçôes dessas magnitudes ocasionam inundaçóes e alagamentos em grandes centros urbanos, devido à impermeabilidade do solo com pavimentaçóes ou similares levando a um maior escoamento das águas. O índice $\mathrm{R} 50 \mathrm{~mm}$ é o mais preocupante, pois representa o número de dias com precipitaçóes iguais ou superiores a $50 \mathrm{~mm}$. Observa-se um aumento com significância estatística desse índice registrado na estaçáo do INMET que está localizada na zona urbana de Manaus. Caso tendências dessa magnitude sejam confirmadas, Manaus sofrerá com o aumento das chuvas extremas, o que pode levar a população às perdas materiais e/ou humanas. Pode ser observado que as outras duas localidades apresentam resultados contrastantes (tendências positivas e negativas) para esse índice, no entanto, não apresentam significâncias estatísticas.

A média anual da precipitação (SDII) apresentou tendência de aumento com significância estatística para as três estaçóes meteorológicas (Figura 2d). No entanto, esse índice não dá um indicativo físico claro, pois, como ele é a razão entre a precipitaçáo total acumulada nos dias úmidos e o número total de dias úmidos no ano, ele pode refletir o aumento na precipitação total anual, como também, uma diminuição do número de dias úmidos. Entretanto, como foi observado que a precipitação total anual apresentou aumento em todas as estaçóes, pode ser conjecturado que esse índice poder indicar uma intensificaçáo dos eventos chuvosos na cidade de Manaus apontando para futuros problemas sociais e econômicos, caso essa tendência de aumento se mantenha. Os índices Dias Consecutivos Secos (DCS) e Dias Consecutivos Úmidos (DCU) concordam com o SDII, pois o DCS apresenta tendência predominante de aumento e o DCU tendência de diminuição. No entanto, ambos os índices não apresentaram significância estatística e, assim, não podem ser associados de forma confiável com os índices discutidos anteriormente.

Os índices Precipitação Máxima Acumulada em 1 dia e em 5 dias consecutivos, Rx1day e Rx5day, respectivamente, mostram tendências predominantes de aumento para as três estaçóes meteorológicas estudadas. No entanto, apenas o índice Rx5day, para a estação do INMET, apresenta tendência com significância estatística. Esse índice representa eventos chuvosos que podem trazer problemas para a população urbana de Manaus, haja vista que o aumento da precipitação acumulada em 5 dias consecutivos pode ser responsável por alagamentos, deslizamentos de encostas e congestionamentos do trânsito numa cidade que tem alta densidade de automóveis nas ruas, entre outros danos.

Os índices que representam os dias úmidos e extremamente úmidos, R95p e R99p, respectivamente, mostram tendência predominante de aumento para a regiâo estudada. Entretanto, apenas o R95p para a estação meteorológica do INMET apresentou tendência com significância estatística. Esses índices corroboram com os demais que indicam tendência de aumento na precipitação sobre a regiáo estudada. Estudos utilizando modelagem, como o de Correia et al. (2007), têm previsto que o desflorestamento da Amazônia pode causar redução na precipitação. Entretanto, esse efeito ainda não é observado para a regiáo de Manaus, conforme os resultados obtidos através dos índices de extremos climáticos analisados, concordando com os encontrados por Satyamurty et al. (2010). Os resultados aqui encontrados concordam com Haylock et al. (2006) que analisaram índices de extremos para algumas localidades da América do Sul, incluindo a estação meteorológica do INMET em Manaus.

A alta variabilidade da precipitação na Amazônia resulta em eventos de completa inundaçáo da bacia amazônica a secas severas (por exemplo, a ocorrida no ano de 2005 e estudada por Marengo et al. 2008). As causas desses eventos têm sido relacionadas aos anômalos aquecimentos e resfriamentos das águas dos Oceanos Pacífico e Attântico. Haja vista que as TSMs são importantes para a manutenção do clima global, especialmente nos trópicos devido à atmosfera ser sensível às condiçôes das superfícies oceânicas e continentais, que influencia grandemente a variabilidade do clima (Yoon e Zeng 2010). Kousky et al. (1984) e Ropelewski e Halpert (1987) mostraram que a precipitaçáo na parte norte da Amazônia e Nordeste do Brasil muda significativamente dependendo da fase do fenômeno ENOS. Recentemente, tem sido documentado que as TSMs do Oceano Atlântico tem um papel na variabilidade do balanço hídrico sobre a América do Sul, influenciando na localizaçáo da Zona de Convergência Intertropical (ZCIT) (Ronchail et al. 2002). Nos últimos anos têm surgido mais evidências de que a influência do Atlântico poderia se estender até a Bacia Amazônica, e a inclusão das TSMs do Atlântico no estudo da Bacia Amazônica pode levar a uma melhor previsão das descargas dos rios sobre a parte mais ao sul da bacia. Assim sendo, é necessário se entender a conexão entre as precipitações na Amazônia e as TSMs do Atlântico, bem como a sua relação com a atividade do ENOS (Yoon e Zeng 2010)

A Tabela 3 apresenta as correlaçôes entre os índices de extremos climáticos dependentes da precipitaçấo diária para a região de Manaus e as anomalias de TSM nos Oceanos Pacífico e Atlântico. Pode-se verificar que as anomalias de TSM na região de Niño 4 apresentam correlação, com significância 
Tabela 3 - Correlações entre os índices de extremos climáticos dependentes da precipitação diária para a região de Manaus e as anomalias de TSM nos Oceanos Pacífico e Atlântico

\begin{tabular}{ccccccc}
\hline & Niño & \multirow{2}{*}{ Niño 3 } & Niño 3.4 & Niño 4 & TNAI & TSAl \\
\hline DCS & $0,386^{*}$ & $0,487^{*}$ & $0,443^{*}$ & $0,293^{*}$ & 0,032 & $-0,479^{*}$ \\
DCU & $-0,167$ & $-0,193$ & $-0,134$ & $-0,101$ & $-0,097$ & $0,307^{*}$ \\
R20mm & $-0,365^{*}$ & $-0,399^{*}$ & $-0,384^{*}$ & $-0,260$ & 0,069 & 0,254 \\
Rx5day & $-0,159$ & $-0,207$ & $-0,245$ & $-0,237$ & $-0,064$ & 0,005 \\
SDII & $-0,175$ & $-0,141$ & $-0,125$ & $-0,021$ & 0,225 & $-0,017$ \\
PRCPTOT & $-0,399^{*}$ & $-0,469^{*}$ & $-0,451^{*}$ & $-0,320^{*}$ & $-0,047$ & $0,404^{*}$ \\
\hline
\end{tabular}

* Indica que o valor do coeficiente de correlação é significativo ao nível de significância de $95 \%(p<0,05)$.

estatística, com os índices DCS e PRCPTOT. Essa região tem correlaçáo positiva com o índice DCS indicando que quando a TSM nessa região aumenta, se observa uma diminuição na precipitação da regiáo amazônica, levando a um aumento do número de dias consecutivos secos. A referida regiáo (Niño 4) tem correlação negativa com o PRCPTOT indicando que quando a TSM da região diminui (fenômeno La Niña) ocorre um aumento na precipitação total anual, mostrando que esses resultados além de apresentarem significâncias estatísticas são concordantes entre si. Correlaçôes negativas foram encontradas para os demais índices, isto é, dias consecutivos chuvosos, número de dias com precipitação acima de $20 \mathrm{~mm}$, o total de precipitaçáo em 5 dias consecutivos, no entanto, não apresentaram significâncias estatísticas. Os resultados encontrados concordam com os obtidos por Yoon e Zeng (2010). As demais regiōes de Niño, isto é, Niño 1+2, 3 e 3.4, também apresentam correlaçôes com significâncias estatísticas com alguns índices, como DCS, R20mm e PRCPTOT. As correlaçôes entre o DCS e as regiôes de Niño 1+2, 3 e 3.4 são positivas indicando que essas regiôes do Pacífico afetam, de forma direta, a distribuição dos dias consecutivos secos sobre a área de estudo, ou seja, um aumento das anomalias de TSM nessas regiōes leva a um aumento dos dias consecutivos secos. As correlaçôes estatisticamente significativas apresentadas entre os índices R20mm e PRCPTOT e as regiôes de Niño $1+2,3$ e 3.4 são todas negativas, também indicando que um aumento nas TSMs dessas regióes leva a uma diminuição dos eventos extremos de chuva e da precipitação total sobre a bacia amazônica, conforme mostrado em Yoon e Zeng (2010).

O índice que representa as anomalias de TSM no Oceano Atlântico Norte (TNAI) não mostrou correlaçôes com significância estatística com nenhum dos índices estudados. Entretanto, sabe-se que um aquecimento do Oceano Atlântico Tropical Norte induz movimento subsidente sobre a bacia amazônica, resultando na diminuição das precipitaçôes na regiâo (Yoon e Zeng 2010), o que pode ser observado, mesmo sem significância estatística, pela correlação negativa entre o TNAI e os índices DCU, Rx5day e PRCPTOT. O oposto pode ser visto na correlação entre o TSAI e os referidos índices, que apresentam correlaçôes positivas (estatisticamente significativas) com DCU e PRCPTOT, indicando que o aquecimento do Oceano Atlântico Tropical Sul induz o deslocamento da ZCIT mais ao sul do Equador levando a um aumento da precipitação sobre a regiáo de Manaus. Isto sugere que a fonte de umidade para a região de Manaus esteja no Atlântico Tropical Sul.

A análise dos índices de extremos para a estação do INMET sugere que existem possíveis efeitos, provenientes $\mathrm{da}$ expansão urbana, na precipitação, pois foi observado aumento com significância estatística para os índices Rx5day, SDII, R50mm e R95p. Ademais, foi observada uma predominância do aumento, embora sem significância estatística, nos demais índices, com exceção do DCS. Pode-se dizer qualitativamente que o efeito de ilha de calor aumenta a temperatura da região urbana que está cercada de rios (laminas de água). Os movimentos verticais positivos sobre a área urbana devem ficar intensificados, causando um provável aumento de precipitação, principalmente na estação do INMET. Então, espera-se que ambos os parâmetros, temperatura e chuva, devem sofrer um aumento por causa da urbanização. Aumento de temperatura deve refletir tanto na temperatura mínima quanto na máxima. No entanto, nesse estudo não foi possível avaliar estes efeitos quantitativamente.

\section{CONCLUSÕES}

Baseado nos resultados discutidos anteriormente pode ser observado que a análise dos índices anuais de extremos climáticos oriundos de dados de precipitação pluvial diária indica aumento da precipitação total anual sobre a região estudada. No entanto, apenas a estação da EMBRAPA apresentou tendência com significância estatística.

$\mathrm{O}$ número de dias no ano com precipitação superior a $10 \mathrm{~mm}$ e $20 \mathrm{~mm}$ aumentou nas estaçóes da EMBRAPA e Reserva Ducke. Eventos de precipitação extrema, isto é, iguais ou superiores a $50 \mathrm{~mm}$, assim como, a precipitação acumulada em um período de cinco dias consecutivos e o número de dias úmidos aumentaram sobre a zona urbana de Manaus. Precipitaçôes dessas magnitudes podem ocasionar inundaçóes e alagamentos em grandes centros urbanos, devido à impermeabilidade do solo com pavimentaçôes ou similares levando a um maior escoamento superficial das águas. Se essa tendência for confirmada, Manaus poderá sofrer com um aumento de enxurradas, o que pode levar a população às perdas materiais e/ou humanas.

Foram constatados através das correlaçôes entre os índices de extremos climáticos dependentes da precipitação diária para a regiáo de Manaus e as anomalias de TSM nos Oceanos Pacífico e Atlântico que aumento das anomalias positivas de TSM nessas regióes leva a um aumento dos dias consecutivos 
secos e a uma diminuição dos eventos extremos de chuva e da precipitação total sobre a bacia amazônica. Foi diagnosticado também que, devido ao aquecimento do Oceano Atlântico Tropical Sul, é induzido o deslocamento da ZCIT mais ao sul do Equador levando a um aumento da precipitação sobre a regiáo de Manaus.

\section{AGRADECIMENTOS}

Os autores agradecem ao CNPq (Conselho Nacional de Desenvolvimento Científico e Tecnológico) pela concessão de bolsas de pesquisa, produtividade e de Pós-Graduação aos autores; ao Centro de Pesquisa Agroflorestal da Amazônia EMBRAPA, a Reserva Florestal Ducke, ao Instituto Nacional de Meteorologia - INMET e ao Climate Prediction Center (CPC) da National Oceanic and Atmospheric Administration (NOAA), por disponibilizarem os dados, assim como, aos pesquisadores Xuebin Zhang e Feng Yang do Serviço de Meteorologia do Canadá, por disponibilizarem gratuitamente o software RClimdex 1.9.0. Finalmente, os comentários dos revisores serviram para melhorar a discussão e as conclusóes.

\section{BIBLIOGRAFIA CITADA}

Aguilar, E.; Aziz Barry, A.; Brunet, M.; Ekang, L.; Fernandes, A.; Massoukina, M.; Mbah, J.; Mhanda, A.; do Nascimento, D.J.; Peterson, T.C.; Thamba Umba, O.; Tomou, M.; Zhang, X. 2009. Changes in temperature and precipitation extremes in western central Africa, Guinea Conakry, and Zimbabwe, 1955-2006. Journal of Geophysical Research, 114, d02115.

Alexander, L.V.; Zhang, X.; Peterson, T.C.; Caesar, J.; Gleason, B.; Klein Tank, A.M.G.; Haylock, M.; Collins, D.; Trewin, B.; Rahimzadeh, F.; Tagipour, A.; Rupa Kumar, K.; Revadekar, J.; Griffiths, G.; Vincent, L.; Stephenson, D.B.; Burn, J.; Aguilar, E.; Brunet, M.; Taylor, M.; New, M.; Zhai, P.; Rusticucci, M.; Vazquez-Aguirre, J.L. 2006. Global observed changes in daily climate extremes of temperature and precipitation. Journal of Geophysical Research, doi: 10.1029=2005JD006290.

Correia, F.W.S.; Alvala, R.C.S.; Manzi, A. 2007. O. Modeling the Impacts of Land Cover Change in Amazonia: A Regional Climate Model (RCM) simulation study. Theoretical and Applied Climatology, 11: 2217-2237.

Fearnside, P.M. 2009. Global warming in Amazonia: Impacts and Mitigation. Acta Amazonica, 39: 1003 - 1012.

Haylock, M.R.; Peterson, T.C.; Alves, L.M.; Ambrizzi, T.; Anunciação, Y.M.T.; Baez, J.; Barros, V.R.; Berlato, M.A.; Bidegain, M.; Coronel, G.; Garcia, V.J.; Grimm, A.M.; Karoly, D.; Marengo, J.A.; Marino, M.B.; Moncunill, D.F.; Nechet, D.; Quintana, J.; Rebello, E.; Rusticucci, M.; Santos, J.L.; Trebejo, I.; Vincent, L.A. 2006. Trends in total and extreme South American rainfall 1960-2000 and links with sea surface temperature. Journal of Climate, 19: 1490-1512.

IBGE, 2009. Instituto Brasileiro de Geografia e Estatística (http:// www.ibge.gov.br/home/mapa_site/mapa_site.php\#populacao). Acesso em 12/12/2009.
Intergovernmental Panel on Climate Change (IPCC). 2007. Climate Change 2007- The Physical Science Basis. Contribution of Working Group I to the Fourth Assessment Report of the IPCC. Cambridge University Press, Cambridge, 2007.

Kousky, V.E.; Cavalcanti, I.F.A.; Kayano, M.T. 1984. A review of the Southern Oscillation: oceanic-atmospheric circulation changes and related rainfall anomalies. Tellus A, 36: 490-504.

Marengo, J.A.; Nobre, C.A.; Tomasella, J.; Cardoso, M.F.; Oyama, M.D. 2008. Hydro-climatic and ecological behaviour of the drought of Amazonia in 2005. Philosophical Transactions of the Royal Society B, 363: 1773-1778.

Menezes, H.E.A.; Brito, J.I.B.; Santos, C.A.C.; Silva, L.L. 2008. A relação entre a temperatura da superfície dos oceanos tropicais e a duração dos veranicos no estado da Paraíba. Revista Brasileira de Meteorologia, 23: 152-161.

Ropelewski, C.; Halpert, M.S. 1987. Global and regional scale precipitation patterns associated with the El Nino-Southern Oscillation. Monthly Weather Review, 115: 1606-1626.

Santos, C.A.C.; Neale, C.M.U.; Rao, T.V.R.; Silva, B.B. 2011. Trends in indices for extremes in daily temperature and precipitation over Utah, USA. International Journal of Climatology, 31: 1813 - 1822 (2011), DOI: 10.1002/joc.2205, 2011.

Satyamurty, P.; Castro, A.A.; Tota, J.; Gularte, L.E.S.; Manzi, A.O. 2010. Rainfall trends in the Brazilian Amazon Basin in the past eight decades. Theoretical and Applied Climatology, 99: 139-148.

Shouraseni, S.R.; Robert, C.B.J.R. 2004. Trends in extreme daily precipitation indices in India. International Journal of Climatology, 24: 457-466.

Viney, N.R.; Bates, B.C. 2004. It never rains on Sunday: The prevalence and implications of untagged multi-day rainfall accumulations in the Australian high quality data set. International Journal of Climatology, 24: 1171-1192.

Yoon, J.H.; Zeng, N. 2010. An Atlantic influence on Amazon rainfall. Climate Dynamics, 34: 249 - 264.

You, Q.; Kang, S.; Aguilar, E.; Pepin, N.; Flugel, W.; Yan, Y.; Xu, Y.; Zhang, Y.; Huang, J. 2010. Changes in daily climate extremes in China and their connection to the large scale atmospheric circulation during 1961-2003. Climate Dynamics, 36:23992417 (2011), DOI: 10.1007/s00382-009-0735-0.

Zhang, Q.; Xu, C.Y.; Zhang, Z.; Chen, X.; Han, Z. 2010. Precipitation extremes in a karst region: a case study in the Guizhou province, southwest China. Theoretical and Applied Climatology, 101: $53-65$.

Zhang, X.; Hegerl, G.; Zwiers, F.; Kenyon, J. 2005. Avoiding inhomogeneity in percentile-based indices of temperature extremes. Journal of Climate, 18: 1641-1651.

Zhang, X.; Yang, F. 2004. RClimDex (1.0) User Guide. Climate Research Branch Environment Canada, Downsview, Ontario, Canada. 22p.

Zin, W.Z.W.; Jamaludin, S.; Deni, S.M.; Jemain, A.A. 2010. Recent changes in extreme rainfall events in Peninsular Malaysia: 1971-2005. Theoretical and Applied Climatology, 99: 303-314.

Recebido em: 14/04/2011

Aceito em: 21/07/2011 
